\title{
Assessment of Quality of Warranty Policy
}

\author{
Dimitar Christozov \\ American University \\ in Bulgaria, \\ Blagoevgrad, \\ Bulgaria \\ dgc@aubg.bg
}

\author{
Stefanka Chukova \\ Victoria University \\ of Wellington, \\ Wellington, New \\ Zealand \\ stefanka@vuw.ac.nz
}

\author{
Plamen Mateev, \\ University of Sofia \\ "St. Kliment \\ Ohridski", Sofia, \\ Bulgaria \\ pmat@fmi.uni-sofia.bg
}

\begin{abstract}
In this study we propose an approach of quantifying the risks of misinforming and malfunctioning aiming to assess the quality of a warranty policy. We identify the "quality" of a warranty policy as an integral measure, based on the balance between two types of warranty - the warranty of malfunctioning and warranty of misinforming. This measure is represented in terms of the length of the warranty coverage, which is the main one-dimensional warranty parameter. A possible approach of identifying the "best" quality warranty policy is outlined.
\end{abstract}

Key words: warranty, information asymmetry, warranty policy, misinforming, malfunctioning, quality

\section{Introduction}

Product warranty is used by the manufacturers/vendors as a mechanism to share the risks associated with the uncertainty of the product performance with their customers. Nowadays warranty is one of the product attributes and it is used as a competing tool on the marketplace. Appropriately assigned product warranty could significantly stimulate the sale process and positively impact the producers' profit. At the same time, the warranty servicing cost should be taken into account, because if these are high enough, they could lead to considerable losses. In Christozov, Chukova, and Mateev (2009a), two types of warranty were identified:

- Warranty of malfunctioning - it is related to product's failure to perform the functions as specified in its description for a predetermined (warranty) period of time $t^{R}=\left[0, t^{R}\right]$, which usually starts right after the sale. The length (size) of the warranty period is usually closely related to reliability and quality of the product. The risk of malfunctioning is shared between the producer and the customer through this warranty of malfunctioning. This type of warranty is widespread and serves many purposes, including protection for producer, seller and consumer.

Material published as part of this publication, either on-line or in print, is copyrighted by the Informing Science Institute. Permission to make digital or paper copy of part or all of these works for personal or classroom use is granted without fee provided that the copies are not made or distributed for profit or commercial advantage AND that copies 1) bear this notice in full and 2) give the full citation on the first page. It is permissible to abstract these works so long as credit is given. To copy in all other cases or to republish or to post on a server or to redistribute to lists requires specific permission and payment of a fee. Contact Publisher@,InformingScience.org to request redistribution permission.
They are used as signals of quality and as elements of marketing strategies. A general treatment of warranty analysis is given by Blischke and Murthy (1993, 1996), Chukova, Dimitrov, and Rykov (1993), Murthy and Djamaludin, (2002), and Karim and Suzuki (2004). From the buyers' point of view, the 
main role of warranty is to provide protection. Specifically the warranty of malfunctioning assures the buyer that a faulty item will either be repaired or replaced at no cost or at a reduced cost. In addition, the warranty is a signal of quality, i.e., it has informational impact on the buyers.

- Warranty of misinforming - it is related to a failure in the communication process during the course of the product sale, which leads to customers being misinformed regarding the product's features and scope of usage. The warranty of misinforming shares the risk of misinforming for a period of time $t^{M}=\left[0, t^{M}\right]$, during which the customers are protected against misinforming, i.e., during this period they can return the product for full reimbursement. This type of warranty is well represented by the slogan "If you are not fully satisfied - money back guaranteed". The concept of information misbalance originates in Arrow (1963), where he introduced it as a "moral hazard." His ideas were further developed by Akerlof (1970), where he investigated the influence of asymmetric information on the market value of a commodity. The impact and usage of the information asymmetry to improve the influence in business relationship branched off from the studies initiated by Akerlof. Slovac (1993) and White and Eiser (2005) studied the asymmetric impact of negative and positive information on the social trust, known as principle of Information Asymmetry or Trust Asymmetry. They concluded that negative information reaches and influences decisions of wider set of potential clients than positive information. Hseih, Lai, and Shi (2006) consider the impact of information asymmetry on the success in business transactions, but they do not go beyond recommendations on how to improve the information process.

Quantifying the risks of malfunctioning of the products is relatively well studied (see Blischke \& Murthy, 1993, 1996). It uses models, based on information obtained from reliability tests, quality control, or other activities carried out by the producer to study product's performance measures. The accuracy of this assessment is entirely under the control of the producer, and the assigned warranty policies fully reflect the objectives of the warrantor. On the other hand, measuring the risk of misinforming is related to failures in the communication processes. It is not under the control of either of the parties involved in the communication, and its evaluation requires different approaches and techniques for collecting and processing data. The primary data source for evaluating the risk of misinforming is the customer's feedback on their satisfaction with the performance and suitability of the product. Christozov, Chukova, and Mateev $(2007,2009 \mathrm{a}, 2009 \mathrm{~b}$, 2009 c) developed a framework for quantifying the risk of misinforming, caused by information asymmetry. Here, by using the proposed measures, we outline a new approach on how to evaluate the "quality" of a given warranty policy.

\section{The Mixed Warranty Policy}

The two types of warranty, the warranty of malfunctioning and warranty of misinforming, have one main parameter of interest - the warranty period. During this period of time ${ }^{1}$, warranty claim against a faulty product is legitimate. Next, based on these two types of warranties, we define a new, so called, mixed warranty policy.

\footnotetext{
${ }^{1}$ Here, by "time" we mean any measure of the warranty coverage, not necessarily the calendar time. For example, in automobile industry, the warranty coverage is identified not only by the vehicle's age, but accounts also for the accumulated mileage, i.e., "time" could by the age of the vehicle, or it could be the accumulated mileage.
} 
Definition 1. A warranty policy, $W_{p}=\left\{W_{R}\left(t^{R}\right) ; W_{M}\left(t^{M}\right)\right\}$, is called a mixed warranty policy, if it accounts for the two types of warranties: $W_{R}\left(t^{R}\right)$ - the warranty of malfunctioning over $t^{R}=\left[0, t^{R}\right]$ and $W_{M}\left(t^{M}\right)$ - the warranty of misinforming over $t^{M}=\left[0, t^{M}\right]$.

The mixed warranty $W_{p}$ is identified by two ordered time periods, $t^{R}$ and $t^{M}$, and we will use interchangeably to the notation in definition 1 , the notation $W_{p}=\left\{t^{R}, t^{M}\right\}$. This definition is an attempt to put together the ideas of warranty of malfunctioning and warranty of misinforming and propose a uniform mechanism for risk sharing for both types of uncertainties - uncertainties related to malfunctioning as well as uncertainties related to misinforming.

\section{Quality of Warranty Policy}

Next, we introduce the notion of quality of warranty policy from producers' as well as from client's viewpoints. Usually, the term "quality" is used to define the complete set of properties pertinent to a given object. From business point of view, quality represents a measure of excellence (see http://www.businessdictionary.com/definition/quality.html). Here, we consider the quality of a mixed warranty policy and identify what represents a high quality warranty policy from producers' as well as from client's viewpoint.

Definition 2. From producers' point of view, the quality, as a measure of excellence, of a mixed warranty policy $W_{p}$, is identified by the expected warranty servicing cost and by the level of client's acceptance of the product.

Definition 3. From producers' point of view, a mixed warranty policy $W_{p}$ is of high (optimal) quality, if it minimizes the expected warranty servicing cost and it maximizes the level of product acceptance by the clients.

These definitions address the two major roles of warranty from the seller's point of view - sharing with the customers the uncertainties of the product performance and promoting the product on the marketplace. Offering no warranty on malfunctioning will reduce the expected warranty cost to zero and will lead to a lower sale price, but at the same time will place the entire risk of malfunctioning or dissatisfaction on the customer. Maximizing the level of acceptance of the product by allowing warranty returns for unlimited time is also unjustified policy from producers' point of view because it could lead to substantial financial losses. A high-quality warranty policy provides a balance between these two extremes. The balance is based on the evaluation of the two risks the risk of malfunctioning and the risk of misunderstanding.

Definition 4. From client's point of view, the quality of a mixed warranty policy $W_{p}$ is identified by the "balanced" value of the "warranty parameter" that provides the best support for the client's correct purchase decision and the level of uncertainty it allows in supporting client's correct purchase decision.

Definition 5. From client's point of view, a mixed warranty policy $W_{p}$ is of high (optimal) quality if the "balanced" value of the "warranty parameter" is maximal and the level of uncertainty it allows in supporting client's correct purchase decision is minimal.

Next, using the models for quantifying the risk of misinforming (Christozov, Chukova, \& Mateev, $2009 \mathrm{~b}$ ) we develop a measure for the quality of a warranty policy from client's point of view. Also, we provide a discussion on the quality of a warranty policy from producers' point of view. 


\section{Quality Comparison between the Mixed and Pure Warranties (Client's Viewpoint)}

We assume that, when making a purchase decision, the client trades off between the following two warranty-related arguments:

- Is the product life cycle long enough? This question is linked to the reliability of the product. The warranty of malfunctioning provides a message related to this question - the longer the warranty of malfunctioning is, the longer the product will preserve its value to the user.

- Is the product suitable for my needs? This question is related to the level of informing during the sale process. The warranty of misinforming encourages clients to examine the product with reduced risk of making a wrong purchase decision.

Let us denote, from client's point of view, the measure of importance of the two warranty parameters by $V_{a}\left(q^{v}, m^{v}\right)$, where $0 \leq q^{v} \leq 1$ is the measure of importance for the reliability parameter and $0 \leq m^{v} \leq 1$ is the measure of importance for the parameter of misinforming. Let assume also that

$q^{v}+m^{v}=1$

i.e., we assume that only these two parameters will influence the client's purchase decision. Another way of looking at (1) is to say that we have imposed a probability distribution on the two warranty parameters.

One possible way of quantifying the quality of a warranty policy $W_{p}=\left\{t^{R}, t^{M}\right\}$ is by assessing how the warranty parameters $t^{R}$ and $t^{M}$ support the clients in making a correct purchase decision. In addition, we assume that an incorrect purchase decision is also unfavorable to the producer/seller, i.e., it increases the warranty servicing cost. This assumption is reasonable because, firstly, the client may not be "fully satisfied" and seek reimbursement and, secondly, s/he may use the product for solving tasks the product is not suitable for, which may result in misuse and within the warranty failure of the product.

Let us denote the set of the warranty parameters by $w:=\left(t^{R}, t^{M}\right)$. So, we have a discrete random variable $w$, which represents the "warranty parameter" with a set of possible values $w:=\left(t^{R}, t^{M}\right)$ and distribution $V_{a}\left(q^{v}, m^{v}\right)$. We make the following interpretation of the expected value $E(w)$ and the standard deviation of $\sigma(w)$ :

- the expected value $E(w)=t^{M} \cdot q^{v}+t^{R} \cdot m^{v}$ represents the "balanced" value of the "warranty parameter" that provides the best support for the client's correct purchase decision. From client's point of view, the "balanced" value $E(w)$ integrates fully the importance of the two warranty aspects - malfunctioning and misinforming. Moreover, increasing the value of the balanced warranty parameter increases the quality of the warranty policy.

- the standard deviation $\operatorname{Var}(w)=\left(t^{M}-E(w)\right)^{2} \cdot q^{v}+\left(t^{R}-E(w)\right)^{2} \cdot m^{v}$ represents the possible error (or deviation) from the best "balanced" value of the warranty parameter. We interpret this deviation as a measure of uncertainty allowed by the policy in supporting client's correct purchase decision. Decreasing the value of this uncertainty increases the quality of the warranty policy. 
Therefore in comparing warranty policies, we assess their quality by comparing their balanced warranty parameters and the measures of uncertainty they allow in supporting a client's correct purchase decision. From the client's viewpoint, the warranty strategy with the largest balanced warranty parameter and the smallest measure of uncertainty is the one with the highest quality. Sometime, in order to identify the best quality warranty policy, a compromise between these two criteria is needed.

Let us compare the expectation of $w$ for the three cases - the two pure warranty strategies and the mixed one. We obtain:

- For the pure warranty of malfunctioning, $W_{p}=\{1,0\}$, we compute that the expected value is

$$
E(w)=1 \times 0.5+0 \times 0.5=0.5,
$$

i.e, the warranty policy supports by $50 \%$ on average the correct decision of the client. The variance is equal to

$$
\operatorname{Var}(w)=\frac{(1.0-0.5)^{2}+(0.0-0.5)^{2}}{2}=0.25 ;
$$

- For the pure warranty of misinforming, $W_{p}=\{0,1\}$, the expected value is

$$
E(w)=0 \times 0.5+1 \times 0.5=0.5
$$

or the warranty policy supports by $50 \%$ on average the correct decision of the client. The variance is equal to

$$
\operatorname{Var}(w)=\frac{(0.0-0.5)^{2}+(1.0-0.5)^{2}}{2}=0.25
$$

- For the mixed warranty $W_{p}=\{0.5,0.5\}$, the expected value is equal to

$$
E(w)=0.5 \times 0.5+0.5 \times 0.5=0.5 \text {, }
$$

i.e., the warranty policy supports by $50 \%$ on average the correct decision of the client. In this case the variance is equal to

$$
\operatorname{Var}(w)=\frac{(0.5-0.5)^{2}+(0.5-0.5)^{2}}{2}=0.0
$$

and the uncertainty of the decisions is equal to zero.

We use this simple example to provide an intuitive illustration for the motivation behind our definitions of quality of warranty policy. We observe that, depending on the length of the warranty periods related to malfunctioning and misinforming, both parameters - expectation and variation - must be considered to distinguish the quality of different policies.

\section{Assessment of the Warranty Policy Parameters}

The two parameters in a mixed warranty policy $W_{p}=\left\{t^{R}, t^{M}\right\}$ represent the warranty coverage with respects of two different risks. Next, we discuss an approach of how to select the "best" values of $t^{R}$ and $t^{M}$ from the producers' point of view. 
Firstly, we focus on the selection of $t^{M}$. Figure 1 represents the clients' learning curve during the warranty (trial) period (see Christozov, Chukova, \& Mateev, 2009c). The trial period starts with an initial level of certainty, $\hat{p}$, on whether the product is suitable to solve his/her tasks and satisfy his/her needs. The assessment of $\hat{p}$ is subjectively made by the client at the time of making the purchase decision. During the trial period, the client's knowledge regarding the actual feature/capabilities of the product to solve his/her tasks and to satisfy his/her needs approaches the real, objective capability of the product, which is estimated to be equal to $p$. The learning time $t$ is proportional to the level of information asymmetry $i a$, which is defined (see Christozov, Chukova, \& Mateev, 2007) as $i a=a b s(\hat{p}-p)$. The larger the information asymmetry the longer learning time needed. If we assume that the shapes of the learning curves of different clients are similar in approaching $p$, the time to reach understanding regarding the suitability of the product depends on the value of the information asymmetry.

In the case of a wrong decision made by an optimist, i.e., $p<q<\hat{p}$, learning means that the assessment regarding capability of the product decreases and crosses the level of acceptance $q$ (as illustrated in Figure 1) at time $t_{c}$. This shape of the learning curves represents our understanding that learning is a logarithmic function on time. For more on the definition/estimation of the level of acceptance $q$ see Christozov, Chukova, and Mateev (2007). At time $t_{c}$, due to the dissatisfaction with the product performance, the client may decide to make a claim against the product. The trial period should be long enough to allow the client to make a claim at time $t_{c}$. For this type of clients extending the trial period will support their purchase decision.

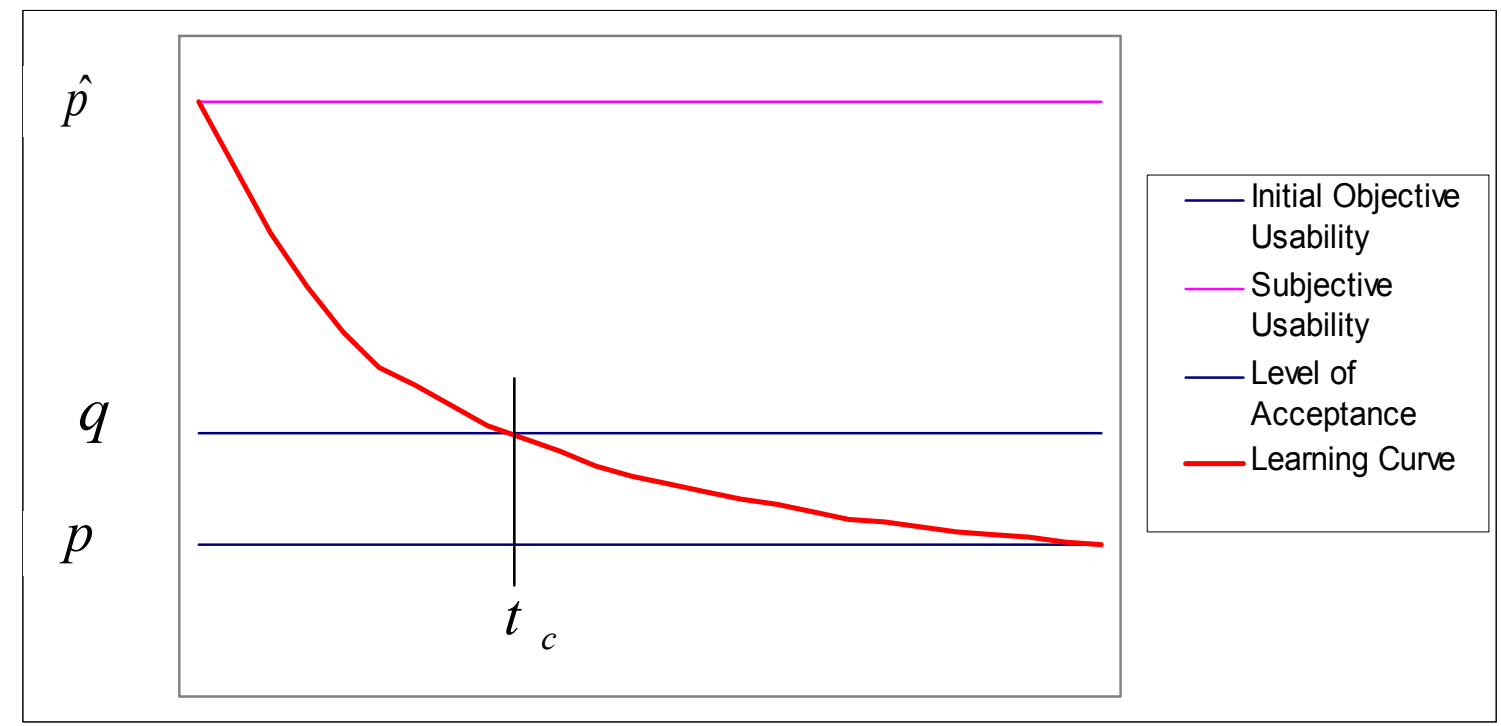

Figure 1. The learning curve during the trial period

In the case of a client who is an optimist but who made a correct decision, i.e., $q<p<\hat{p}$, as well as for all other types of clients - pessimists and realists, extending the trial period will lead only to extra time of using the product potentially for free and the warranty servicing cost may be unnecessary increased. 
In general, the parameter $t^{M}$ has to be selected according to the proportion of optimists in the clients' population, the level of their risk of misinforming, and the level of corresponding information asymmetry.

The second parameter $t^{R}$ represents the warranty coverage related to malfunctioning of the product. It is based on the evaluation of the length of the three sub-periods within the product's life cycle:

- Decreasing Failure Rate sub-period (DFR) - early failures due to manufacturing defects, (infant mortality);

- The sub-period of a constant failure rate;

- Increasing Failure Rate sub-period (IFR) - failures caused by product's increasing age, consumption, or use.

A typical curve representing the three periods of failure rate, is shown in Figure 2.The parameter $t^{R}$ definitely has to cover the DFR sub-period or $t^{R}>t_{0}$ and in addition it should such that the inequality $t^{R}<t_{1}$ is valid. The failure rate in the interval $\left[t_{0}, t_{1}\right]$ is a constant and the warranty cost will be proportional to its length. So overall, we would like to minimize the length of $t^{R}$. On the other hand, the promotional strength of the warranty policy is also proportional to the length of $t^{R}$ and we would like to maximize this strength, which is equivalent of maximizing $t^{R}$. For example, selecting $t^{R}=t_{1}$ may give certain competitive advantages. Therefore, it is essential to find a value of $t^{R}$ that assures the best balance between these two opposite trends.

\section{Failure Rate}

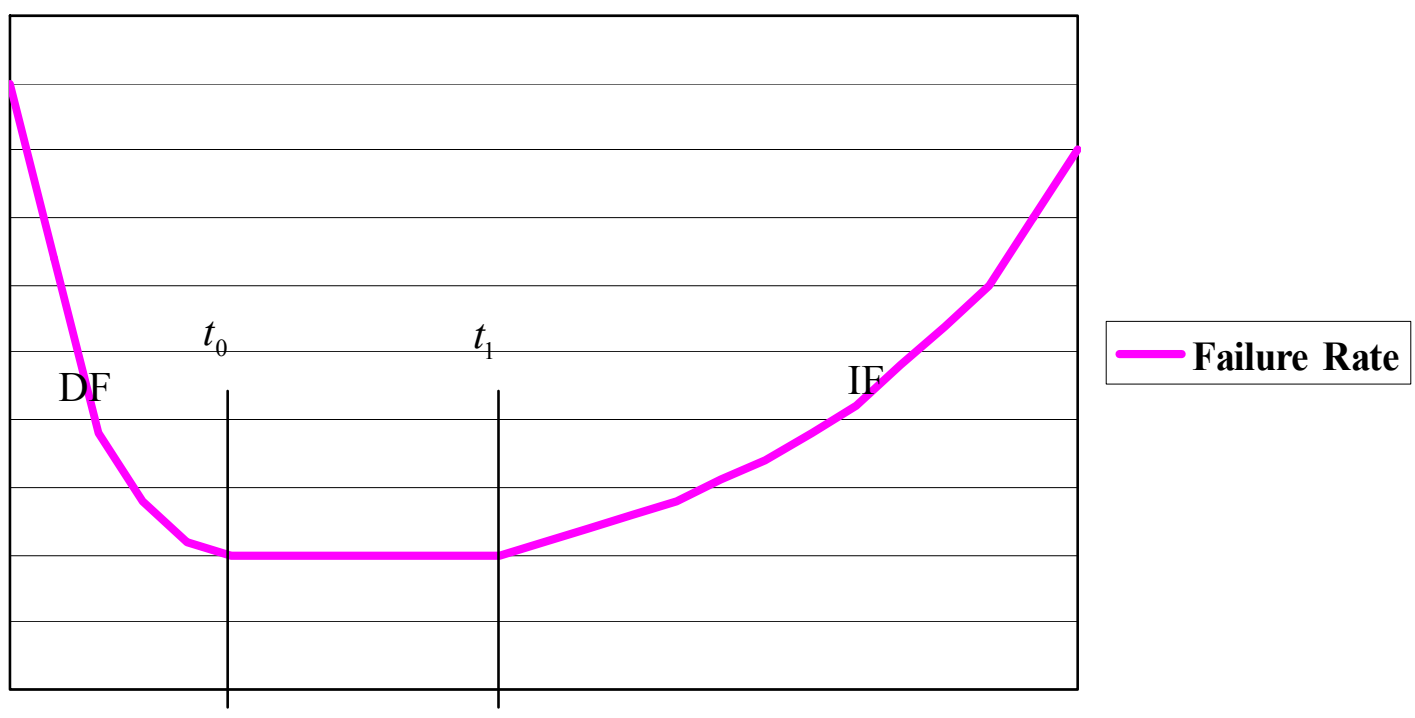

Figure 2. Failure rate function over product's life cycle

Onwards, we will assume that the "best" value of $t^{R}$ is known. Further comments on the issue of selecting $t^{R}$ are given later, in our concluding remarks. In addition, the determination of the parameter of misinforming $t^{M}$ needs a careful study of clients' population and their risk of misinforming. In the next section we use the measures of the risk of misinforming to identify the notion 
of the "quality" of a warranty policy from the producers' point of view. We illustrate the ideas using an example drawn from our previous empirical studies.

\section{Quality of Warranty Policy as a Function of the Risk of Misinforming}

We will use data from a survey conducted at Victoria University of Wellington, Wellington, New Zealand in 2006. For more on the survey design see Christozov, Chukova, and Mateev (2007). Here, we will use only one of the two data samples collected in this survey - the data sample for the feedback of the group of respondents who had a choice between three possible warranty strategies. This sample consists of 54 data points. The survey was designed and conducted to collect information regarding parameters, utilized by the client in his/her purchasing decision making process. We were interested on the responses regarding:

- the needs $n_{i j}$ for solving a particular task;

- the level of acceptance $q_{i j}$;

- the indicator of a wrong decision $r_{i j}$;

- the level of asymmetry $i a_{i j}$.

Also, every respondent in the survey selected a preferred warranty policy among the three possible options:

- three year of pure warranty of malfunctioning;

- three months of pure warranty of misinforming;

- $\quad$ mixed warranty with parameters $t^{R}=$ one year and $t^{M}=$ one month.

We use the survey information and consider the following cases, summarized in Table 1. In Table $1, W_{i}\left(t^{R}, t^{M}\right)$ denotes the warranty policy for clients with risk level $i$, where $i=\{$ low, medium, high\}.

Table 1. Map of warranty policies

\begin{tabular}{|l|l|l|l|l|}
\cline { 2 - 4 } \multicolumn{2}{c|}{} & \multicolumn{3}{c|}{ Correctness of the purchase decision } \\
\cline { 2 - 5 } \multicolumn{2}{c|}{} & \multicolumn{1}{c|}{ Low risk } & \multicolumn{1}{c|}{ Medium risk } & \multicolumn{1}{c|}{ High risk } \\
\cline { 2 - 5 } & $r_{j}>r_{u}$ & $r_{l} \leq r_{j} \leq r_{u}$ & $r_{j}<r_{l}$ \\
\hline \multirow{3}{*}{$\begin{array}{l}\text { Preferred war- } \\
\text { ranty policy }\end{array}$} & $W_{p}(36,0)$ & $W_{l}(36,0)$ & $W_{m}(36,0)$ & $W_{h}(36,0)$ \\
\cline { 2 - 5 } & $W_{p}(0,3)$ & $W_{l}(0,3)$ & $W_{m}(0,3)$ & $W_{h}(0,3)$ \\
\cline { 2 - 5 } & $W_{p}(12,1)$ & $W_{l}(12,1)$ & $W_{m}(12,1)$ & $W_{h}(12,1)$ \\
\hline
\end{tabular}

In Table $1, r_{j}$ denotes any of the three measures of the risk of misinforming defined in Christozov, Chukova, and Mateev (2009b) by formulae (1), (2) and (3). For the reader's convenience these formulae are provided in the Appendix of this report. The values of the bounds of the risk of 
misinforming - the lower bound $r_{l}$ and upper bound $r_{u}$ are selected, so they represent suitably the range of this risk for the respondents.

The ratios of respondents falling in each of the above nine categories will allow us to assess the quality of the warranty policy with respect to each particular target group. The average adjusted risk for the group of 54 respondents was evaluated at 0.296 . Within this group the minimum computed risk was 0.030 and the maximum computed risk was equal to 0.573 . We consider the range $[0,0.6]$ of the risk, and define the lower and upper bounds uniformly across the range as $r_{l}=0.2$ and $r_{u}=0.4$. Using the survey data and the selected values for the risk boundaries, we have estimated the entries of Table 1 and presented the results in Table 2.

Table 2. Data, according 2006 survey

\begin{tabular}{|c|c|c|c|c|c|c|c|c|c|c|}
\hline & \multicolumn{8}{|c|}{ Correctness of the purchase decision } \\
\hline & & & \multirow{2}{*}{\multicolumn{2}{|c|}{$\begin{array}{l}\text { Low risk } \\
r_{j}<0.2\end{array}$}} & \multirow{2}{*}{\multicolumn{2}{|c|}{$\begin{array}{c}\text { Medium risk } \\
0.2_{l} \leq r_{j} \leq 0.4\end{array}$}} & \multirow{2}{*}{\multicolumn{2}{|c|}{$\begin{array}{l}\text { High risk } \\
r_{j}>0.4\end{array}$}} & \multirow{2}{*}{\multicolumn{2}{|c|}{ Total }} \\
\hline & & & & & & & & & & \\
\hline & & & No & $\%$ & No & $\%$ & No & $\%$ & No & $\%$ \\
\hline \multirow{4}{*}{\multicolumn{2}{|c|}{$\begin{array}{l}\text { Preferred } \\
\text { policy }\end{array}$}} & $W_{p}(36,0)$ & 7 & 20.5 & 19 & 56.0 & 8 & 23.5 & 34 & 63.0 \\
\hline & & $W_{p}(0,3)$ & 0 & 0.0 & 0 & 0 & 1 & 100.0 & 1 & 2.0 \\
\hline & & $W_{p}(12,1)$ & 6 & 31.6 & 12 & 63.2 & 1 & 5.2 & 19 & 35.0 \\
\hline & & Total & 13 & 24.0 & 31 & 57.4 & 10 & 18.6 & 54 & 100 \\
\hline
\end{tabular}

Table 2 shows that for 34 of the respondents, the corresponding distribution of importance is $V_{a}(1,0)$ and they selected $W_{p}(36,0)$. For one of the respondents, who selected $W_{p}(0,3)$, the corresponding distribution of importance is $V_{a}(0,1)$ and for the remaining 19 respondents, who selected the mixed warranty strategy $W_{p}(12,1)$, we assume that the corresponding distribution of importance is $V_{a}(0.5,0.5)$. Based on this information, using the approach sketched in the section on Assessment of the Warranty, the quality of the warranty strategies, from the client's point of view, could be evaluated.

The group of respondents is dominated by the sub-group of optimists - they are 38 out of 54 and the average risk of misinforming for this group is 0.337 . This value is close to the upper bound of the medium level risk range. The other two sub-groups of respondents are the sub-group of pessimists ( 4 out of 54), with the average risk of misinforming 0.311 , and the sub-group of realists (12 out of 54), with the average risk of misinforming 0.159 . The average value of information asymmetry for the whole group is 0.19 , with information asymmetry computed only for the subgroup of optimists equal to 0.24 .

To assess the quality of the three warranty policies, from a producer point of view, we will apply the following criteria:

1. Assess whether the policy supports the client's purchase decision making process. Looking at the above data, we may consider that the pure strategy $W_{p}(0,3)$ serves less than two percent of the population in their decision making process. Obviously, this strategy 
cannot meet this quality criterion. The strategies $W_{p}(36,0)$ and $W_{p}(12,1)$ are supporting decision of significant number of respondents.

2. Assess the effect of the offered warranty on the reputation of the seller. The cost of misinforming on the reputation of the seller/producer with the first pure warranty strategy is defined as the proportion of unsatisfied clients who cannot claim full reimbursement on their product. For the above data, only $20 \%$ of clients will be "fully satisfied" (low risk), $56 \%$ will experience medium dissatisfaction, and $23 \%$ will be extremely disappointed by their purchase. Therefore, nearly $80 \%$ of the customers will experience significant dissatisfaction, which may have a considerable negative impact on the reputation of the seller. Thus, the pure strategy $W_{p}(36,0)$ cannot be considered as a high quality warranty policy.

Based on the above discussion, we conclude that the data obtained in our empirical study supports our intuition that the mixed warranty policy is of higher quality, from the producer's point of view, than the two pure warranty policies.

\section{Conclusion}

In this study we propose an initial model for identifying the notion of "quality of a warranty policy." We introduce the notion of mixed warranty policy to combine the effect of warranty of malfunctioning and warranty of misinforming. In addition, we identify the quality of a warranty policy from two different viewpoints: client's viewpoint as well as producer's point of view. We propose an initial approach of quantifying the quality of warranty policy from the client's viewpoint. Using our previous results and empirical survey data, we present a discussion on the quality of several warranty options. The expectation that the mixed warranty strategy outperforms any of the pure warranty strategies motivated this study. It is more at conceptual level attempting to identify the concept of "quality" of a warranty policy and to define approaches and criteria to measure and compare different policies. At the same time, during the course of this study, we have identified a list of open problems that need further research. For example, an open problem that deserves a close attention is how to identify the "best" mixed warranty strategy mathematically: should this identification be formulated as an optimization problem; should some constraints be imposed on the warranty parameters; should the parameters of a mixed warranty strategy be considered as independent or should some dependence be introduced; etc. In this study we used data collected earlier, i.e., for different purposes, to illustrate our new ideas. Our next task will be to design an experiment for collecting appropriate empirical data, which allow a better illustration and application of our new models related to the quality of warranty policy.

\section{References}

Akerlof, G. A. (1970). The market for 'lemons': Quality uncertainty and the market mechanism. Quarterly Journal of Economics, 84(3), 488-500.

Arrow, K. (1963). Uncertainty and the welfare economics of medical care. The American Economic Review, 53(5), 941-973.

Blischke, W., \& Murthy, D.N.P. (1993). Warranty cost analysis. New York: Marcel Dekker.

Blischke, W., \& Murthy, D.N.P. (1996). Product warranty handbook. New York: Marcel Dekker.

Christozov, D., Chukova, S., \& Mateev, P. (2007). On the relationship between warranty and the risk of information asymmetry. Journal of Issues in Informing Science and Information Technology, 4, 235249.

Christozov, D., Chukova, S., \& Mateev, P. (2009a). On two types of warranties: Warranty of malfunctioning and warranty of misinforming. Asia-Pacific Journal on Operation research, 26(3), 399-420. 
Christozov, D., Chukova, S., \& Mateev, P. (2009b) Informing processes, risks, evaluation of the risk of misinforming. In T. G. Gill \& E. Cohen (Eds.), Foundations of informing science (pp. 323-356). Santa Rosa, CA: Informing Science Press.

Christozov, D., Chukova, S., \& Mateev, P. (2009c). The risk of misinforming for competing messages. Journal of Issues in Informing Science and Information Technology, 6, 351-364. Retrieved from http://iisit.org/Vol6/IISITv6p351-364Christozov627.pdf

Chukova, S., Dimitrov, B., \& Rykov, V. (1993). Warranty analysis. A survey. Journal of Soviet Mathematics, 67(6), 3486-3508.

Hseih, C-T., Lai, F., \& Shi, W. (2006). Information orientation and its impact on information asymmetry and e-business adoption: Evidence from China's international trading industry. Industrial Management \& Data Systems, 106(6), 825-840.

Karim, R., \& Suzuki, K. (2004). Analysis of warranty claim data: A literature review. In W. Yun \& T. Dohi (Eds.), Proceedings of the 2004 Asian International Workshop on Advanced Reliability Modeling (pp. 229-237). Japan: World Scientific.

Murthy, P. D., \& Djamaludin, I. (2002). New product warranty: A literature review. International Journal of Production Economics, 79(2), 236-260.

Slovac, P. (1993). Perceived risk, trust, and democracy. Risk Analysis, 13, 675-682.

White, M., \& Eiser, R. (2005). Information specificity and hazard risk potential as moderators of trust asymmetry. Risk Analysis, 25(5), 1187-1198.

\section{Appendix. Measures for the Risk of Misinforming}

(for more details see Christozov, Chukova, \& Mateev, 2009b)

A simple model - only data regarding the risk of wrong decisions $r_{i j}$ is available. The proposed measure of the risk of misinforming is:

$$
r_{j}^{s}=\frac{1}{I} \sum_{i=1}^{I} r_{i j}
$$

A model with known needs $n_{i j}$ and risks of wrong decisions $r_{i j}$. We have proposed the following measure for the risk of misinforming:

$$
r_{j}^{n}=\frac{1}{I} \frac{1}{\sum_{i=1}^{I} n_{i j}} \sum_{i=1}^{I} n_{i j} r_{i j}
$$

A model with known $\left(r_{i j}, n_{i j}, i a_{i j}\right)$ - the risks of wrong decisions $r_{i j}$, the needs $n_{i j}$ and the information asymmetry $i a_{i j}$. The proposed measure of the risk of misinforming is:

$$
r_{j}^{a}=\frac{1}{I} \frac{1}{\sum_{i=1}^{I} n_{i j}} \sum_{i=1}^{I} n_{i j} r_{i j} i a_{i j} .
$$




\section{Biographies}

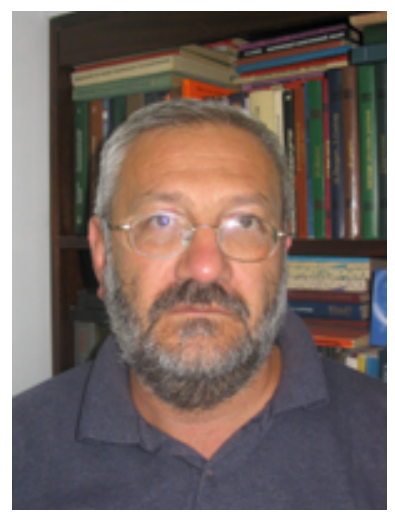

Dimitar Christozov is a Professor of Computer Science at the American University in Bulgaria, Blagoevgrad 2700, Bulgaria since 1993, email: dgc@aubg.bg. He has more than 30 years of experience in areas such as computer science, quality management, and information systems. He graduated Mathematics from Sofia University "St. Kliment Ohridski" in 1979. He completed his PhD thesis "Computer Aided Evaluation of Machine Reliability" in 1986. In 2009 he completes his D.Sc. thesis on "Quantitative Measures of Informing Quality". In ICTT "Informa" (1986-1993). Dr. Christozov was involved in establishing the national information network for technology transfer and research in the areas of technologies assessment, integral quality measures and information systems for quality management. In these areas he was recognized as one of the leading experts in Bulgaria. Professor Christozov has more than 70 publications as separate volume, journal papers, and papers in refereed proceedings. He is a founding member of Informing Science Institute and chair of Bulgarian Informing Science Society; and member of the Bulgarian Statistical Society.

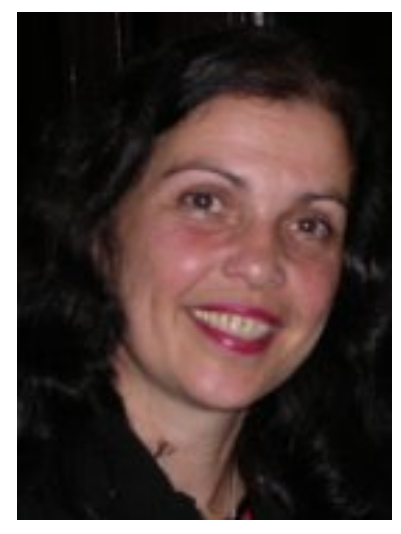

Dr. Stefanka Chukova is an Associate professor in Statistics and Operations Research at the School of Mathematics, Statistics and Operation Research, Victoria University of Wellington, Private Bag 600, Wellington, New Zealand, e-mail: schukova@mcs.vuw.ac.nz. She has a PhD and MSc in Mathematics (concentration in Probability and Statistics) and BSc in Mathematics from University of Sofia, Sofia, Bulgaria. Her research interests are in applied stochastic models, warranty analysis, reliability and queuing. She has more than 50 publications and has presented papers at national and international conferences. She is a member of ORSNZ, AWIS and ASA.

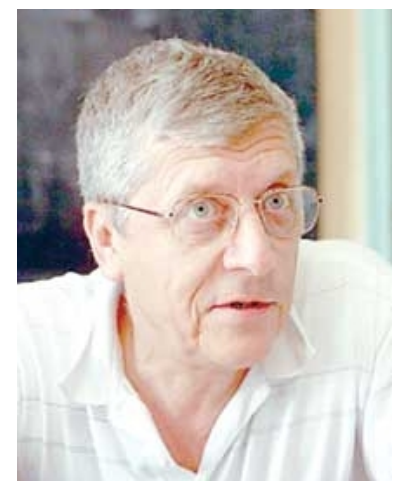

Dr.Plamen S. Mateev is Associate professor in Faculty of Mathematics and Informatics, Sofia University "St.Kliment Ohridski", Department "Probability, Operation Research, Statistics", Bulgaria, 1164 Sofia, 5, J.Boucher str., e-mail: pmat@fmi.uni-sofia.bg.

His MSc in Mathematical Statistics is from Sofia University and his $\mathrm{PhD}$ is from Moscow State University. The research interests are in communication theory, applied statistics, statistical software and applications. More than 70 papers are published in scientific journals and proceedings of scientific conferences. He is the Chair of Bulgarian Statistical Society and member of ENBIS and Bulgarian Informing Science Society. 